\title{
Transpleural ventilation of explanted human lungs
}

\author{
Cliff K Choong, Peter T Macklem, John A Pierce, Stephen S Lefrak, Jason C Woods, Mark S Conradi, \\ Dimitry A Yablonskiy, James C Hogg, Kimiaki Chino, Joel D Cooper
}

Thorax 2007;62:623-630. doi: 10.1136/thx.2005.053256

See end of article for authors' affiliations

Correspondence to:

Dr Joel D Cooper, Hospital of the University of

Pennsylvania, 3400 Spruce St, 6 Silverstein,

Philadelphia, Pennsylvania

19104,USA; joel.cooper@ uphs.upenn.edu

Received 23 September 2005 Accepted 8 December 2006

\begin{abstract}
Background: The hypothesis that ventilation of emphysematous lungs would be enhanced by communication with the parenchyma through holes in the pleural surface was tested.

Methods: Fresh human lungs were obtained from patients with emphysema undergoing lung transplantation. Control human lungs were obtained from organ donors whose lungs, for technical reasons, were not considered suitable for implantation. Lungs were ventilated through the bronchial tree or transpleurally via a small hole communicating with the underlying parenchyma over which a flanged silicone tube had been cemented to the surface of the lung (spiracle). Measurements included flow-volume-time curves during passive deflation via each pathway; volume of trapped gas recovered from lungs via spiracles when no additional gas was obtainable passively from the airways; and magnetic resonance imaging assessment of spatial distribution of hyperpolarised helium $\left({ }^{3} \mathrm{He}\right)$ administered through either the airways or spiracles.

Results: In emphysematous lungs, passively expelled volumes at $20 \mathrm{~s}$ were $94 \%$ greater through spiracles than via the airways. Following passive deflation from the airways, an average of 1.07 litres of trapped gas volume was recoverable via spiracles. Regions were ventilated by spiracles that were less well ventilated via bronchi.

Conclusions: Because of the extensive collateral ventilation present in emphysematous lungs, direct communication with the lung parenchyma through non-anatomical pathways has the potential to improve the mechanics of breathing and hence ventilation.
\end{abstract}

$\mathrm{H}$ yperinflation and gas trapping are devastating consequences of severe emphysema. The increase in total lung capacity (TLC) compromises chest wall mechanics as the thorax approaches the limit of expansion. Gas trapping increases the residual volume (RV) to TLC ratio and reduces both the slow and forced vital capacity (VC and FVC, respectively). This in turn is a major cause of reduction in forced expiratory volume in $\mathrm{l} s\left(\mathrm{FEV}_{1}\right)$. The progressively narrowing gap between RV and TLC increases the work of breathing and continues to decrease ventilatory capacity until the patient becomes flow-limited even while breathing at rest. Thus, a major objective of treatment must be to decrease the amount of trapped gas.

Collateral ventilation in emphysema greatly exceeds that in normal lungs, as demonstrated in lungs removed at necropsy by Hogg et al in 1969, ${ }^{1}$ confirmed using a different technique in patients by Terry et al in $1978^{2}$ and reconfirmed more recently by Morrell et al. ${ }^{3}$ Macklem, in an editorial comment, stated: “... if collateral flow resistance is less than airway resistance ... ventilation ... through openings directly through the chest wall into the parenchyma should bypass the obstruction, decrease work of breathing, increase alveolar ventilation and improve dyspnea. Insects breathe through openings on their body surface ... called spiracles." ${ }^{\prime 4}$

Lausberg et al recently reported that the creation of fistulas between segmental bronchi and adjacent lung parenchyma increased $\mathrm{FEV}_{1}$ and FVC in explanted emphysematous human lungs. ${ }^{5}$ The present study was designed to test the hypothesis that ventilation of low resistance collateral pathways in emphysema via spiracles through the pleura will allow trapped gas to escape and increase passive flow and volume on deflation. Openings were made directly into the parenchyma through tubes glued to the pleural surface (spiracles) in order to measure flow and volume that traversed the spiracles. Emphysematous lungs explanted from lung transplant recipients were compared with nearly normal lungs obtained from donors but rejected for transplantation because of minor abnormalities.

\section{METHODS}

Preliminary studies on four emphysematous lungs showed the feasibility of studying ventilation through transpleural communications. This allowed us to develop a protocol by which we measured: (1) volume-flow-time relationships during passive deflation through either the bronchial tree or the spiracles from full inflation; (2) the amount of trapped gas that could be removed via the spiracles after the lung was completely deflated passively through the airways; and (3) the distribution of ventilation as assessed by MRI scans of hyperpolarised helium $\left({ }^{3} \mathrm{He}\right)$. The complete protocol was successfully carried out in two control lungs ( 1 and 2 ) from one donor whose lungs for technical reasons were rejected for implantation and from a consecutive series of five left lungs (3-7) explanted from five patients (three women and two men) with end-stage emphysema undergoing double lung transplant surgery. In the preliminary studies we did not complete all three parts of the protocol in any of the four lungs. We report the complete data from lungs 1-7 and, where appropriate, limited measurements on the lungs from the preliminary studies.

The mean values of preoperative pulmonary function studies are shown in table 1. The spiracle (Hood Laboratories, Pembroke, Massachusetts, USA) made of medical grade silicone $(1.7 \mathrm{~mm}$ thick) was a quasi-elliptical tube $(9 \times 18 \mathrm{~mm}$ externally) with a flange $(33 \times 21 \mathrm{~mm})$ that was glued with tissue cement to the lung as shown in fig 1 . The pleura was incised through the lumen of the tube and a $3 \times 10 \mathrm{~mm}$ area of

Abbreviations: $\mathrm{CL}$, lung compliance; $\mathrm{FEV}_{1}$, forced expiratory volume in $1 \mathrm{~s}$; FVC, forced vital capacity; Gaw, airway conductance; Gsp, spiracle conductance; $\mathrm{PA}$, alveolar pressure; Pel, elastic recoil pressure; Pfr, pressure producing flow; Ppl, pleural pressure; ROI, region of interest; RV, residual volume; TLC, total lung capacity; VC, vital capacity 
Table 1 Mean and standard deviation (SD) values from preoperative pulmonary function studies

\begin{tabular}{llllll}
\hline & FVC & FEV $_{\mathbf{1}}$ & RV & TLC & Raw (cm/l/s) \\
\hline Mean, I (\%) & $1.64(46 \%)$ & $0.42(15 \%)$ & $6.4(314 \%)$ & $8.3(147 \%)$ & $4.92(273 \%)$ \\
SD, I (\%) & $0.70(13 \%)$ & $0.17(6 \%)$ & $1.9(77 \%)$ & $2.7(33 \%)$ & $2.2(111 \%)$ \\
\hline
\end{tabular}

FVC, forced vital capacity; FEV 1 , forced expiratory volume in $1 \mathrm{~s}$; RV, residual volume; TLC, total lung capacity; Raw, airway resistance; $\%$, percentage of predicted normal.

pleura was removed. The lung parenchyma was thus accessed directly through the lumen of the spiracle. Spiracles were attached to both upper and lower lobes in each emphysematous lung while the control lungs each had only one spiracle.

Each main stem bronchus was securely fitted with a cannula. The lung was inflated to TLC as judged by visual inspection with a series of small volume bursts of pressure delivered manually from a compressible respirator bag. Aside from this, only gentle massage and repeated filling/emptying cycles with airway pressure limited to $20 \mathrm{~cm} \mathrm{H}_{2} \mathrm{O}$ were used to achieve full inflation. A major difficulty in studying the mechanical properties of emphysematous lungs ex vivo is air leakage. To circumvent this problem, major vessels were ligated to avoid leakage from the vascular and perivascular spaces. Small tears or cuts on the surface were identified by immersion in isotonic saline solution and repaired by focal ligation or tissue cement. When the lung was considered fully inflated, the bronchial
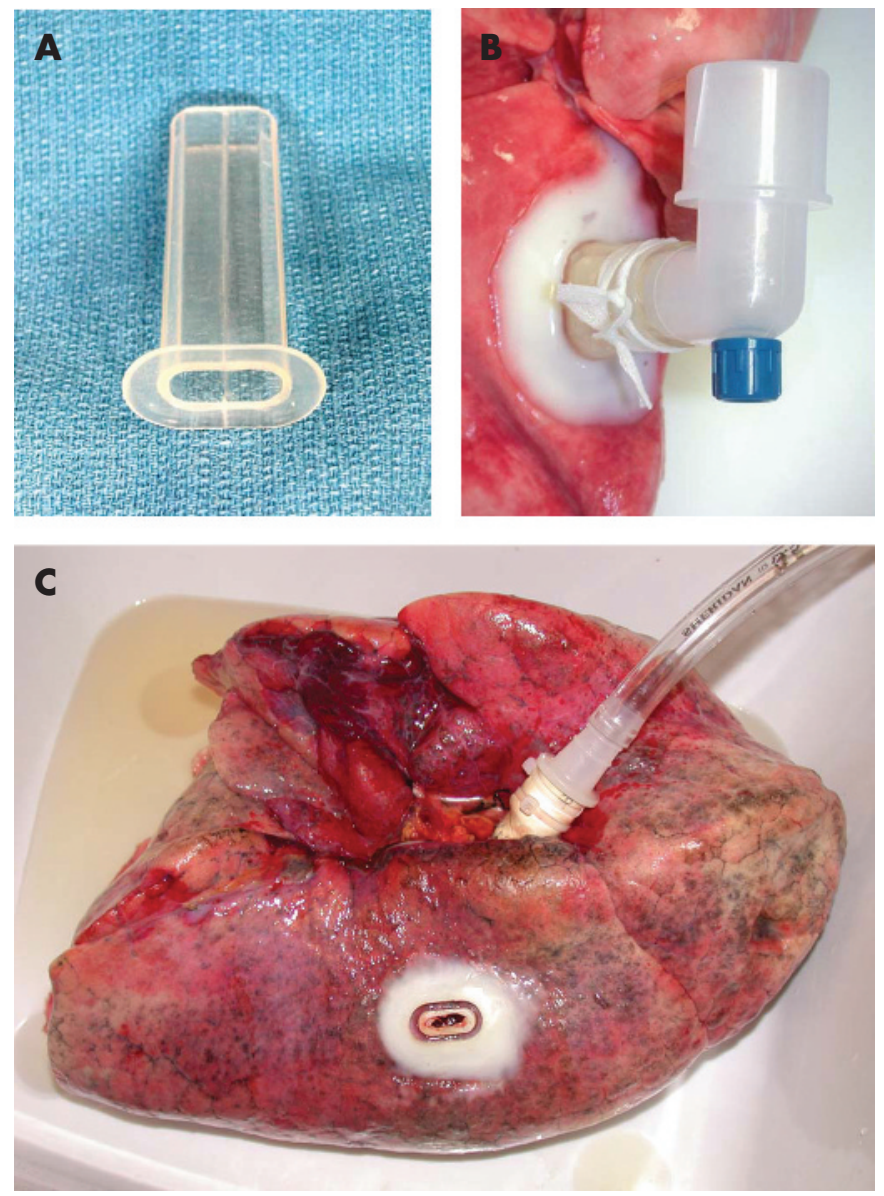

Figure 1 Transpleural spiracle. (A) Quasi-elliptical silicone tube with a $33 \times 21 \mathrm{~mm}$ flange. (B) Device secured to the pleura with tissue cement. A segment of pleura was excised through the lumen of the tube to permit communication with the lung parenchyma. (C) Site of transpleural communication following removal of the silicone spiracle tube. cannula was opened and the lung was allowed to deflate passively through the airways.

With the spiracles closed, the lung was re-inflated via the bronchus to transpulmonary pressures of 8.1-15.1 $\mathrm{cm} \mathrm{H}_{2} \mathrm{O}$ in the emphysematous lungs ( $14.0-20.6 \mathrm{~cm} \mathrm{H}_{2} \mathrm{O}$ in control lungs) and allowed to deflate passively via the airway ${ }^{6}$ through a pneumotachometer (RSS100-HR, Sensor Model 3700, HansRudolph, Kansas City, Missouri, USA; flow range 0-160 1/min that includes airway and auxiliary pressure transducers) with commercial software (RSS100-HR Research Pneumotach System Version 3.0.7b; KORR Medical Technologies, Salt Lake City, Utah, USA). The lung was again re-inflated to a roughly similar pressure $\left( \pm 1 \mathrm{~cm} \mathrm{H}_{2} \mathrm{O}\right)$ through the bronchus. The bronchial cannula was then closed and flow and volume were measured during passive deflation through the spiracle(s). At least three passive flow-volume curves were obtained through each pathway and the results were averaged. We also measured the volumes expelled passively at 1, 5, 10, 15, and $20 \mathrm{~s}$ and beyond, to or near completion through each pathway.

The lung was then fully re-inflated through the bronchus (to a pressure of $10-15 \mathrm{~cm} \mathrm{H}_{2} \mathrm{O}$ ), the bronchus was opened and the lung allowed to expel as much air as possible by passive deflation through the airways. The time was not recorded but commonly exceeded $1 \mathrm{~min}$ and uniformly exceeded $30 \mathrm{~s}$. The bronchial cannula was then clamped and the spiracle(s) were opened, allowing gas trapped behind closed airways to exit passively through the spiracles into a Mylar bag. A Y-connector was used to collect gas from both lobes simultaneously in the emphysematous lungs. The volume of trapped gas expelled was measured from the bag into a calibrated syringe.

The breath by breath distribution of ventilation of hyperpolarised ${ }^{3} \mathrm{He}$ (two control and five emphysematous lungs) was compared by MRI. ${ }^{7}$ Hyperpolarised ${ }^{3} \mathrm{He}$ of nominal polarisation $40 \%$ was prepared with two locally constructed and one commercial polariser (Amersham Health, Chalfont St Giles, Buckinghamshire, UK). To prevent ${ }^{3} \mathrm{He}$ depolarisation by contact with oxygen, we first ventilated the lung with $100 \%$ nitrogen for $20 \mathrm{~min}$ using an open circuit technique and stored the lung in an airtight plastic bag containing 100\% nitrogen. With intrabronchial pressure set at $2-3 \mathrm{~cm} \mathrm{H}_{2} \mathrm{O}$, a tidal volume of approximately $300 \mathrm{ml}$ of hyperpolarised ${ }^{3} \mathrm{He}(\mathrm{P} \geqslant 40 \%)$ was instilled via a gas syringe to either the bronchus or spiracle. The lung was tidally ventilated by closed circuit rebreathing with the same volume for up to 10 breaths. Images were obtained at the end of each inflation cycle on a Siemens Magnetom Vision whole body scanner at $48.47 \mathrm{MHz}\left({ }^{3} \mathrm{He}\right.$ frequency at a nominally 1.5-T field). A home-built, four-turn, in-parallel, solenoid-like coil with high Q took advantage of the lack of saline and low radio frequency loss.

Following the last ${ }^{3} \mathrm{He}$ deflation-inflation cycle, $300 \mathrm{ml}$ of lung gas were removed, replaced with $300 \mathrm{ml}$ room air from the syringe, and open circuit washout of ${ }^{3} \mathrm{He}$ imaging was performed for up to six cycles. ${ }^{3} \mathrm{He}$ imaging was repeated at the end of each inflation. Regions of interest (ROI) were chosen in each lobe near where the spiracles were located, to represent a macroscopic gas exchange volume that was significantly more efficient when delivered via spiracles than via the bronchus. 

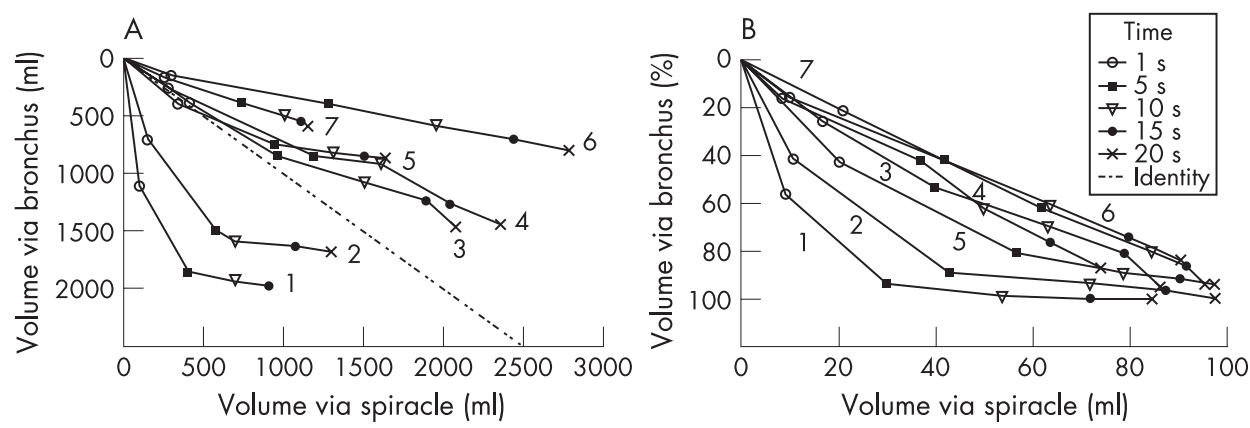

Figure 2 (A) Isotime identity plot of passive deflation from full lung inflation (control pressure $\sim 20 \mathrm{~cm} \mathrm{H}_{2} \mathrm{O}$, emphysema pressure $\sim 10 \mathrm{~cm} \mathrm{H}$ O). The absolute volume expelled from the bronchial tree is plotted on the ordinate against the absolute volume expelled from the spiracle on the abscissa at 1, 5, 10, 15, and $20 \mathrm{~s}$ after the onset of passive deflation. Numbers near the final data points identify each lung. (B) Same data plotted on similar coordinates as in (A) as a percentage of the total volume expelled.

These ROI were hand drawn to encompass a region approximately 0.5 litres in volume in each lobe and generally encompassed three image slices. The total ROI signal (equivalent to the total magnetic moment in the region and thus the total volume of ${ }^{3} \mathrm{He}$, absent the minimal effects of $\mathrm{T}_{1}$ here) was integrated and fit to a linear function. After the first extraction of $300 \mathrm{ml}$ gas and subsequent insertion of $300 \mathrm{ml}$ room air, the ROI signal was again integrated.

\section{Analysis of data}

Mean values are presented with standard deviation (SD). Differences in mean values were analysed with the Student's $t$ test. ${ }^{9}$ Time constants were calculated for the mean volume-time curves of each lung with the equations: Vexp,aw(t) = Vexp,aw $[1-\exp (-\mathrm{t} / \mathrm{T}, \mathrm{aw})]$ or $\operatorname{Vexp}, \mathrm{sp}(\mathrm{t})=\operatorname{Vexp}, \mathrm{sp}[1-\exp (-\mathrm{t} /$ Tau,sp)] using the software Systat Version 11 (Systat Software Inc, Richmond, California, USA) where $\operatorname{Vexp}, \mathrm{aw}(\mathrm{t})$ and Vexp,sp(t) are the volumes expelled via each route at each time point (t); Vexp,aw and Vexp,sp are the total volumes expelled by each route; exp is e, the base of the natural system of logarithm; $-\mathrm{t}$ is the time in seconds to total volume; and Tau is the time constant. The values for $R^{2}$ were all 0.97 or greater.

\section{RESULTS}

A critical determinant of lung inflation is mean airway pressure. For this series of measurements in emphysematous lungs, mean (SD) initial pressure was 10.7 (2.6) $\mathrm{cm} \mathrm{H}_{2} \mathrm{O}$ $(\mathrm{n}=30)$. For the bronchial curves it was $10.2(2.3) \mathrm{cm} \mathrm{H}_{2} \mathrm{O}$ $(\mathrm{n}=15)$ and for the spiracle curves $11.3(2.9) \mathrm{cm} \mathrm{H}_{2} \mathrm{O}(\mathrm{n}=15)$. While this is perhaps more variation than ideal, it is evenly distributed between the two groups of observations and is unlikely to have distorted the results. Inflation pressures for the control lungs averaged $18.8 \mathrm{~cm} \mathrm{H}_{2} \mathrm{O}(\mathrm{n}=12)$. Curves for the bronchial route averaged $20.5(2.8) \mathrm{cm} \mathrm{H}_{2} \mathrm{O}(\mathrm{n}=6)$ and for the spiracle pathway $17.1(3.9) \mathrm{cm} \mathrm{H}_{2} \mathrm{O}(\mathrm{n}=6)$. Non-linear regression was performed according to the equation described in the Methods section in order to obtain time constants (Tau) for the mean volume-time curves of each lung.

Figure 2 compares volumes passively expelled from the bronchus and spiracles of lungs inflated as described above. In fig $2 \mathrm{~A}$ the volumes are given as absolute values. In the control lungs ( 1 and 2 ) the rates and volumes expelled via the bronchus greatly exceeded the results via spiracles and the time constants were much shorter. In the emphysematous lungs (37) the absolute volume of gas expelled from the spiracles usually exceeded the rate and volume observed via the bronchus (table 2). Table 2 also shows the mean initial airway pressure for the three curves studied and the length of time considered complete to total volume. These results varied from 26 to $47 \mathrm{~s}$ in the emphysematous lungs. Even so, explanted emphysematous lung airways continue to expel air (although slowly) beyond these periods. The mean length of time to total volume of the emphysematous lungs was 36 s via the bronchial route and $34 \mathrm{~s}$ via the spiracle(s). The mean (SD) total gas volume expelled from the emphysematous lungs at $20 \mathrm{~s}$ via the spiracle(s) was $194(61) \%$ of that via the bronchus $(p=0.01$, $\mathrm{n}=5)$.

Figure 2B shows the volumes normalised as a percentage of the total volume expelled. The data points are on (lungs 6 and 7) or close to (lungs 3 and 4) the line of identity for all

Table 2 Passively expelled volumes in $\mathrm{ml}$

\begin{tabular}{|c|c|c|c|c|c|c|c|c|}
\hline Lung number & $\begin{array}{l}\text { Mean initial } \\
\text { airway pressure* } \\
\left(\mathrm{cm} \mathrm{H}_{2} \mathrm{O}\right)\end{array}$ & $1 \mathrm{~s}$ & $5 \mathrm{~s}$ & $10 \mathrm{~s}$ & $15 \mathrm{~s}$ & $20 \mathrm{~s}$ & $\begin{array}{l}\text { Final total } \\
\text { volume (ml) }\end{array}$ & $\begin{array}{l}\text { Time to } \\
\text { total volume }(s)\end{array}$ \\
\hline \multicolumn{9}{|c|}{ Bronchial pathway } \\
\hline 1 & 20.3 & 1102 & 1853 & 1941 & 1974 & & 1974 & 15 \\
\hline 2 & 20.6 & 700 & 1488 & 1588 & 1631 & 1681 & 1681 & 20 \\
\hline 3 & 12.2 & 390 & 826 & 1080 & 1235 & 1460 & 1528 & 42 \\
\hline 4 & 9.6 & 260 & 696 & 1035 & 1267 & 1444 & 1669 & 28 \\
\hline 5 & 9.8 & 400 & 750 & 822 & 853 & 870 & 926 & 26 \\
\hline 6 & 8.1 & 153 & 400 & 582 & 704 & 800 & 953 & 37 \\
\hline 7 & 10.8 & 131 & 390 & 503 & 544 & 596 & 632 & 47 \\
\hline \multicolumn{9}{|c|}{ Spiracle pathway } \\
\hline 1 & 14.0 & 128 & 446 & 793 & 1070 & 1258 & 1476 & 30 \\
\hline 2 & 20.2 & 150 & 574 & 960 & 1163 & 1295 & 1327 & 30 \\
\hline 3 & 15.1 & 410 & 962 & 1507 & 1895 & 2074 & 2384 & 38 \\
\hline 4 & 8.1 & 280 & 1190 & 1603 & 2044 & 2361 & 3205 & 44 \\
\hline 5 & 10.1 & 340 & 944 & 1308 & 1509 & 1636 & 1662 & 32 \\
\hline 6 & 9.4 & 300 & 1283 & 1950 & 2444 & 2783 & 3049 & 25 \\
\hline \multirow{2}{*}{\multicolumn{9}{|c|}{ All airways }} \\
\hline & & & & & & & & \\
\hline 7 & 14.4 & 469 & 978 & 1125 & 1175 & 1197 & 1200 & 25 \\
\hline
\end{tabular}


Table 3 Time constants (Tau) in seconds

\begin{tabular}{lccl}
\hline $\begin{array}{l}\text { Lung } \\
\text { number }\end{array}$ & $\begin{array}{l}\text { Bronchial } \\
\text { route }(\mathbf{s})\end{array}$ & $\begin{array}{l}\text { Spiracle } \\
\text { route }(\mathbf{s})\end{array}$ & $\begin{array}{l}\text { All } \\
\text { airways (s) }\end{array}$ \\
\hline 1 & 1.3 & 12.1 & \\
2 & 2.0 & 7.8 & \\
3 & 7.2 & 9.6 & \\
4 & 9.9 & 14.0 & \\
5 & 2.4 & 6.0 & \\
6 & 10.3 & 9.3 & 2.5 \\
7 & 5.8 & 5.2 & \\
Mean & 7.1 & 9.3 & \\
SD & 3.3 & 3.5 & \\
\hline
\end{tabular}

emphysematous lungs except lung 5. Thus, in lungs 4,6 and 7, the time constants for emptying were roughly similar for both pathways while in lung 5 , which emptied faster through the bronchial tree than it did through the spiracles, the time constant for the bronchial pathway was much smaller than that for the spiracles. This was confirmed by the Tau values shown in table 3. In the control lungs the Tau values were 9.3 and 3.9 times longer for emptying through the spiracles than through the airways. Lung 5 had the shortest Tau for emptying via the airways and the largest percentage difference between airway and spiracle Tau values for the emphysematous lungs; this lung showed an airway emptying pattern close to normal (fig 2B). In the emphysematous lungs the difference between the two Tau values varied from $10 \%$ to $60 \%$ of the larger value. Figure 3 shows additional examples of flow-volume and volume-time curves passively expelled.

Table 4 shows the volume of gas recovered from upper and lower lobe spiracles simultaneously after full inflation and complete passive airway deflation of the emphysematous lungs. The mean volume was 1.07 litres (range $0.21-1.57$ ). The fact that the trapped gas in the emphysematous lungs was expelled passively through the spiracles indicates that it was under positive pressure, that is, small airways close and trap gas prematurely when alveolar pressure is still positive. This gas did not communicate with the bronchial cannula when the lung was fully deflated. Table 4 also shows the recovery of trapped gas RV from five upper and five lower individual lobe spiracles: mean 0.69 litres (range $0.18-1.41$ ), $\mathrm{n}=10$. During the preliminary studies in one explanted emphysema lung, 1.49 litres of trapped gas was recovered from one spiracle; a preliminary report was published as an abstract. ${ }^{10}$

Figure 4 shows the breath by breath distribution of ${ }^{3} \mathrm{He}$ in a slice of a control left lung (lung 1) and a slice of an emphysematous left lung (lung 6). In each panel the left hand column shows the ${ }^{3} \mathrm{He}$ distribution for breaths 1,3 and 5 via the bronchial tree whereas the right columns illustrate the distribution for the same breath numbers via the spiracles. In the control lungs the distribution of ${ }^{3} \mathrm{He}$ gas was similar by both routes. This contrasts with the distribution of ${ }^{3} \mathrm{He}$ in the explanted emphysematous lung. When ventilation occurred via the airways, most of the ${ }^{3} \mathrm{He}$ entered the left lower lobe. The sharp line of demarcation extending toward the left and slightly downward is caused by the major fissure. Immediately above the fissure, the upper lobe remained essentially unventilated. The apex of the upper lobe received a small amount of ${ }^{3} \mathrm{He}$ but, after five breaths, the entire upper lobe remained very poorly ventilated compared with the lower lobe. In contrast, when ventilation took place simultaneously via spiracles in both lobes, the distribution of ${ }^{3} \mathrm{He}$ was more even. After five breaths, only regions bordering the fissure remained poorly ventilated and, even there, more ${ }^{3} \mathrm{He}$ entered through the spiracles than through the bronchial tree. Figure 4 shows that (1) trapped gas regions accessed by spiracles tend to be large (size of segments and even lobes); (2) within them the ventilation distribution was reasonably uniform; and (3) gas was distributed via the spiracles to regions that were not as well ventilated by the bronchus. Although each emphysematous lung differed from the others in terms of regional heterogeneity, all lungs showed these three features (fig 4). This indicates that spiracles can provide low resistance pathways for ventilatory flow to much of the emphysematous lung.

Similarly, tidal ventilation with room air using an open circuit technique via spiracles produced large areas of gas depletion that corresponded to the bright regions seen earlier on initial ${ }^{3} \mathrm{He}$ inflation. ROI were selected near sites of spiracle placement to quantify gas depletion of specific volume. The mean ROI volume of $467 \mathrm{ml}$ was depleted by an average of $55 \%$ of ${ }^{3} \mathrm{He}$ on the first cycle. In contrast, only $9 \%$ of ${ }^{3} \mathrm{He}$ was depleted in the ROI after a single cycle via the bronchial tree. Detailed results are shown in table 5 and fig 5 .
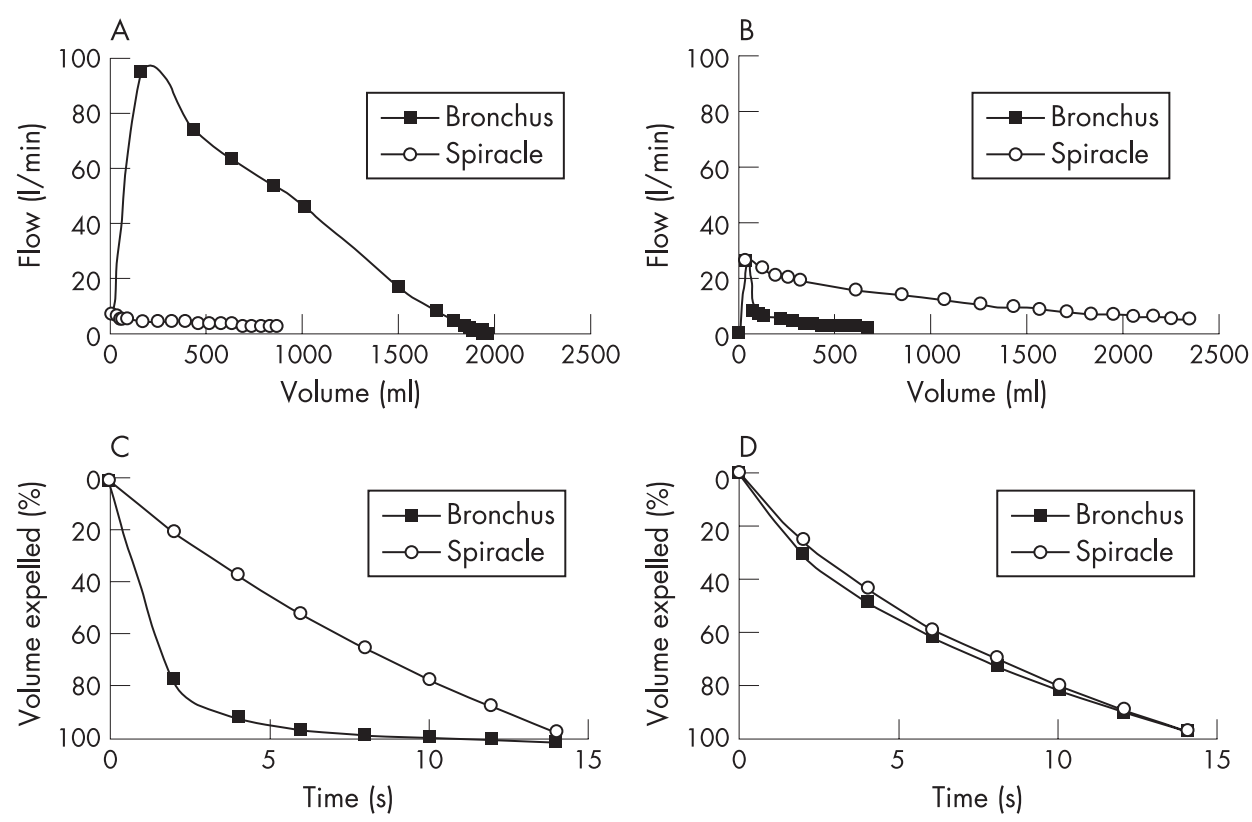

Figure 3 (A, B) Flow-volume curves and (C, D) percentage volume-time curves during passive emptying (free collapse). Data points appear every $0.2 \mathrm{~s}$ for the first second and each second thereaffer to $15 \mathrm{~s}$. (A) Flowvolume curve recorded from a near normal lung (lung 1) with bronchial flow greatly exceeding spiracle flow. (B) Representative tracing from the lung of a patient with severe pulmonary emphysema (lung 6) showing passively expelled flow and volume via the spiracle exceeding that via the bronchial tree. (C, D) A different perspective of the same data in which volume on the ordinate is expressed as a percentage of the total volume expelled passively versus time. In (D) it can be seen that, when volume is expressed in this way, the pattern of emptying from the emphysematous lung via the airways and spiracles is nearly identical, indicating that the time constant for emptying is similar for both routes. 
Table 4 Volume of trapped gas recovered from emphysematous lungs

\begin{tabular}{llll}
\hline Lung number & $\begin{array}{l}\text { (A) Volume recovered } \\
\text { simultaneously from } \\
\text { two spiracles (I) }\end{array}$ & $\begin{array}{l}\text { (B) Volume recovered } \\
\text { from single upper } \\
\text { lobe spiracle (I) }\end{array}$ & $\begin{array}{l}\text { (C) Volume recovered } \\
\text { from single lower } \\
\text { lobe spiracle (I) }\end{array}$ \\
\hline 3 & 1.14 & 1.31 & 0.89 \\
4 & 1.57 & 0.60 & 0.51 \\
5 & 1.04 & 0.54 & 0.69 \\
6 & 1.41 & 0.56 & 1.41 \\
7 & 0.21 & 0.25 & 0.18 \\
Mean & $1.07^{*}$ & $0.65^{*}$ & $0.74^{*}$ \\
SD & 0.53 & 0.39 & 0.46 \\
\hline *Not statistically significantly different. & \multicolumn{3}{l}{} \\
Column A is the gas recovered via two spiracles simultaneously after full lung inflation and complete passive deflation via \\
the bronchus. Columns B and C are the volumes of trapped gas recovered from single upper and lower lobe spiracles \\
respectively after full lung inflation and complete passive deflation via the bronchus.
\end{tabular}

\section{DISCUSSION}

Laennec ${ }^{11}$ described the handling of an emphysematous lung as that perceived when handling a pillow of down. Hogg et al ${ }^{1}$ suggested this sensation due to the ease with which air can be pushed from one area of an emphysematous lung to another. Thoracic surgeons are familiar with the fact that emphysematous lungs remain inflated after excision, except when the presence of a small puncture or tear in the pleura results in complete collapse. This feature can be attributed to the extensive collateral ventilation in emphysema. Can one imagine any way of destroying the delicate parenchyma of the lung without causing an increase in collateral ventilation?

Previous measurements of collateral ventilation in excised emphysematous lungs have shown that the resistance to collateral flow across an incomplete major fissure is less than the resistance to flow through the airways, whereas in normal lungs the opposite occurs. ${ }^{12}$ The first measurements in living patients with emphysema performed by Terry et al ${ }^{2}$ confirmed low resistance of collateral ventilatory pathways. Morrell et al ${ }^{3}$ devised an ingenious bronchoscopic method to demonstrate a functionally important correlation between increased collateral ventilation and increased oxygen tension in the peripheral lung parenchyma. When the patient (or subject) inspired heliox (79\% helium, $21 \%$ oxygen), the concentration of helium distal to a blocked peripheral bronchus rose 10 times faster in patients with moderately severe emphysema than in those with healthy lungs. Compared with healthy lungs, emphysematous lungs regularly exhibit increased collateral ventilation.

Higuchi et al $^{13}$ recently reported interlobar collateral ventilation in 15 of 23 explanted emphysematous lungs. The authors cannulated and ventilated the bronchus to one lobe and achieved active ventilation of a companion lobe that was not cannulated. They analysed the preoperative results of CT scans and V/Q scintigraphs in a carefully blinded manner to classify the emphysema of each lung as homogeneous or heterogeneous. Interlobar collateral ventilation was almost uniformly present in the lungs of patients with homogeneous emphysema while only $40 \%$ of patients with heterogeneous emphysema exhibited significant interlobar collateral ventilation. In an accompanying editorial, Cetti $e t$ al $^{14}$ suggested that the range of anatomical changes in emphysema must be more complicated than the two categories allowed. The authors also pointed out that interlobar ventilation must be an advanced stage of collateral ventilation which is almost certainly a continuous variable in the spectrum of emphysema. Our group correlated the long range diffusion of hyperpolarised ${ }^{3} \mathrm{He}$ to morphometric measurements of lung surface area/volume ratios in normal and emphysematous lungs by measuring the decay of spatially

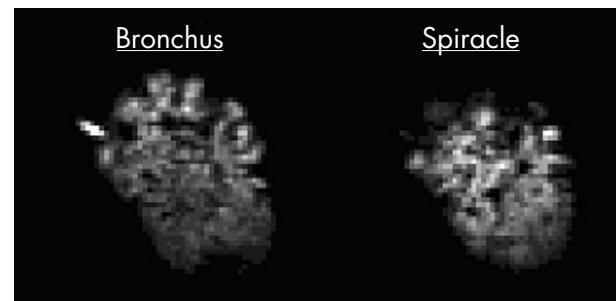

Breath \# 1

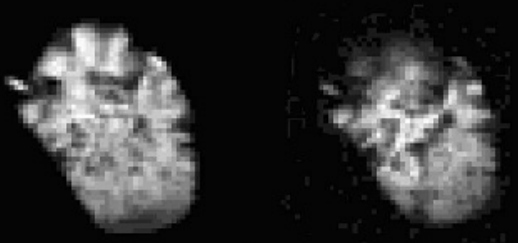

Breath \#3

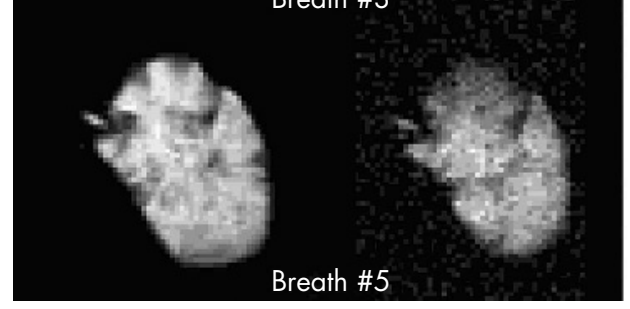

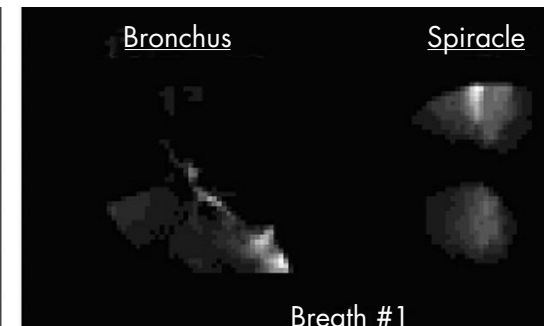

Breath \#1

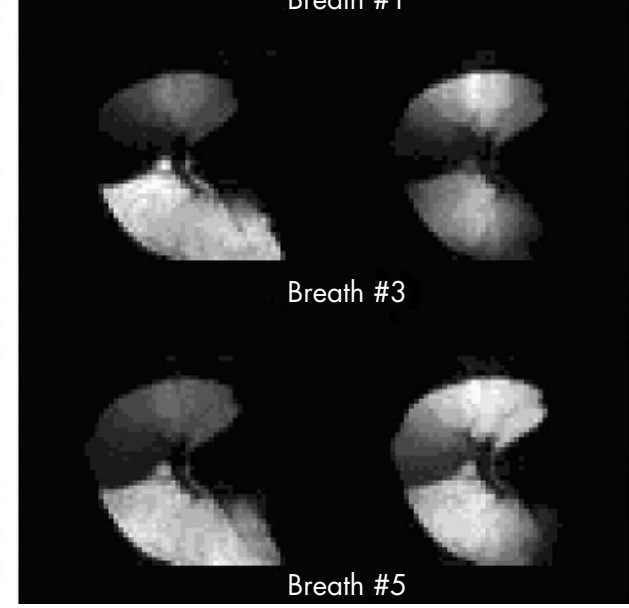

Figure 4 Magnetic resonance images of the distribution of hyperpolarised helium $\left({ }^{3} \mathrm{He}\right)$ ventilation via the bronchus and spiracles in a healthy left lung (lung 1) with one spiracle (left panel) and an emphysematous left lung (lung 6) with spiracles in both upper and lower lobes (right panel). The light areas depict the location of the hyperpolarised ${ }^{3} \mathrm{He}$. Images made following the initial inflation presented at the top are followed by images after the third and fifth breaths, respectively, in the centre and lower panels. The distribution of ventilation was similar via the bronchus and via one spiracle in the healthy lung. The emphysematous lung, however, showed preferential ventilation of the lower lobe via the bronchus, and large dense areas of rather uniform ventilation around the area of the spiracles during sequential breaths. 


\begin{tabular}{|c|c|c|c|c|}
\hline Lung number & Lobe & ROI volume (ml) & Bronchus (\%) & Spiracle (\%) \\
\hline \multirow[t]{2}{*}{3} & U & 522 & 9.8 & 18 \\
\hline & L & 430 & 0.2 & 28 \\
\hline \multirow[t]{2}{*}{4} & U & 629 & 0 & 52 \\
\hline & L & 476 & 0 & 69 \\
\hline \multirow[t]{2}{*}{5} & U & 450 & 0 & 55 \\
\hline & L & 329 & 20 & 59 \\
\hline \multirow[t]{2}{*}{6} & U & 373 & 24 & 44 \\
\hline & L & 560 & 0 & 73 \\
\hline \multirow[t]{2}{*}{7} & U & 520 & 33 & 78 \\
\hline & L & 385 & 8 & 74 \\
\hline Mean & & 467 & $9.5^{*}$ & $55^{*}$ \\
\hline SD & & & 12 & 20 \\
\hline
\end{tabular}

modulated longitudinal ${ }^{3} \mathrm{He}$ magnetisation. ${ }^{15}$ This technique assesses collateral ventilation with a greater sensitivity than that of interlobar ventilation. We agree with Cetti et $a l^{14}$ that understanding collateral ventilation is important for planning bronchoscopic methods of treatment for patients with advanced emphysema.

The lungs used in the present study were typical of those from patients undergoing transplantation for emphysema at our centre (table 1). Mean values of $\mathrm{FEV}_{1}$ of $15 \%$ and $\mathrm{RV}$ of $314 \%$ of predicted normal clearly demonstrate severe expiratory flow limitation with marked hyperinflation characteristic of advanced emphysema. The subjects in the present study had more severely impaired preoperative pulmonary function than those in the group studied by Morrell et al. ${ }^{3}$ Dramatically increased collateral ventilation can be safely predicted in the lungs used in the present study, but it remains to be seen whether the functional benefits observed would also be found in lungs with less severe functional abnormalities or in living patients.

\section{Main finding}

The main finding of this study is that transpleural ventilation via spiracles communicates with areas of the parenchyma that do not communicate with the trachea at RV. As a result, our hypothesis that the introduction of spiracles would decrease trapped gas, increase VC and expiratory flows proved to be correct. Measured preoperatively, the mean RV in this series of patients with emphysema was 6.40 litres. We recovered $17 \%$ of this amount (1.07 litres) via transpleural spiracles after the completion of passive bronchial deflation from one lung. While the amount might seem small, it approaches one third of RV for both lungs and would be highly significant for a patient with chronic obstructive pulmonary disease.

\section{Pathophysiology of emphysema}

We believe that our results shed interesting new information on the functional abnormalities of emphysema that warrants a detailed consideration of the pathophysiology. Lung mechanics have been modelled for several decades using electrical analogues. In these models, capacitors represent the elastic properties of the lung and its compliance $\left(C_{L}\right)$, whereas resistors are analogous to the flow resistance of the airways. Expressed as conductances, the airway conductance (Gaw) and the spiracle conductance (Gsp) sum (Gaw + Gsp) to express the total conductance when breathing takes place through both pathways simultaneously. The time constant for emptying of the lung through the tracheobronchial tree is then given by $\mathrm{CL} /$ Gaw, while that for emptying through the spiracle is CL/Gsp. If emptying occurs through both pathways, the time constant is given by $\mathrm{CL}_{\mathrm{L}} /(\mathrm{Gaw}+\mathrm{Gsp})$. Alveolar pressure (PA) is the pressure
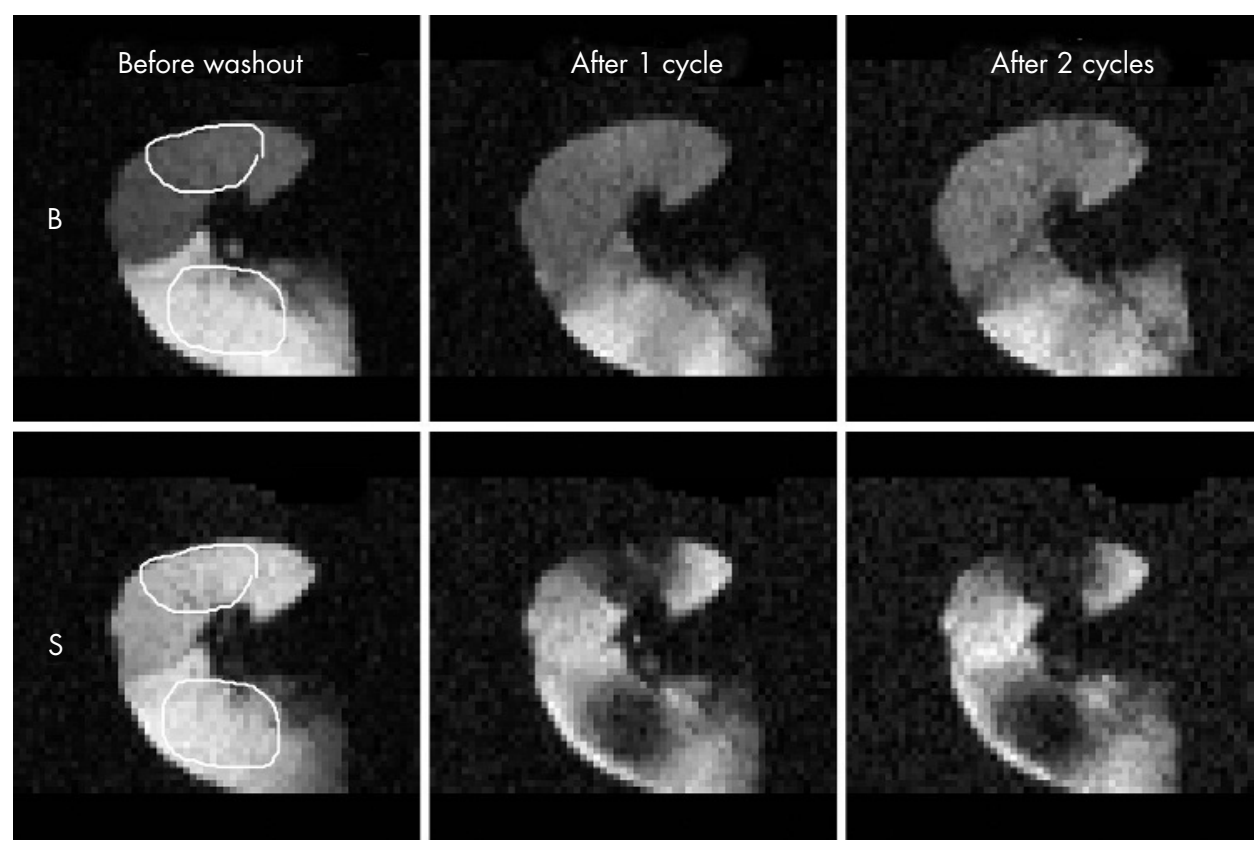

Figure 5 Washout helium $\left({ }^{3} \mathrm{He}\right)$ images of lung 5 via bronchial (B) and spiracle (S) pathways. The left panels show distribution of the gas following the final closed circuit ${ }^{3} \mathrm{He}$ deflation/inflation (300 ml) cycle with the selected regions of interest (ROI) outlined in white. The centre panels show the result following one open circuit deflation/inflation $(300 \mathrm{ml})$ cycle with room air. The right panels show the result following a second room air deflation/inflation washout cycle. The striking reduction in intensity during the first cycle is represented as a percentage of total signal depletion in ROI for lung 5 in this figure. Data for all the emphysematous lungs are shown in table 5 . 

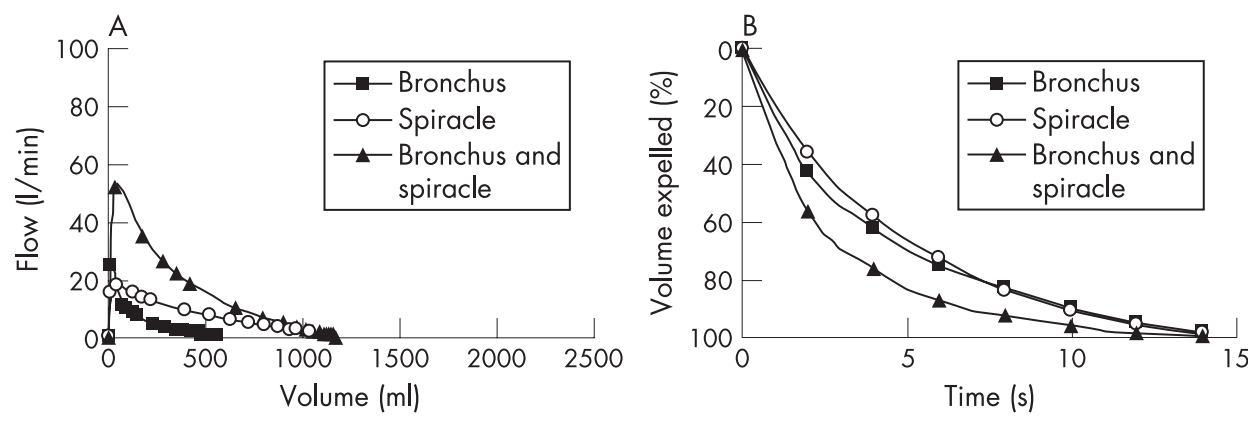

Figure 6 (A) Flow-volume curve and (B) percentage volume-time curve of passive collapse obtained from the lung of a patient with severe emphysema (lung 7). The percentage volume-time relations were similar for passive emptying through the bronchus and via the spiracles, indicating similarity in emptying times via both routes. However, when emptying occurred through both the airways and spiracles simultaneously, the emptying time was shortened. There was therefore a reduction in the time constant for passive emptying when this occurred through the spiracles and airways simultaneously (see text). producing flow (Pfr) and is analogous to the voltage across the resistors. PA relative to pleural pressure $(\mathrm{Ppl})$ or lung elastic recoil pressure $(\mathrm{PA}-\mathrm{Ppl}=\mathrm{Pel})$ is analogous to the voltage across the capacitors. When $\mathrm{Ppl}$ is atmospheric (as in our experiments), Pfr and Pel are identical. Furthermore, Pfr is common to both airways and spiracles and the resulting flow is the product of $\mathrm{Pfr}=\mathrm{PA}=\mathrm{Pel}$ and the appropriate conductance. If one assumes that a patient with severe emphysema has a Gaw of $0.1 \mathrm{l} / \mathrm{s} / \mathrm{cm} \mathrm{H}_{2} \mathrm{O}$ and a Gsp of $0.3 \mathrm{l} / \mathrm{s} / \mathrm{cm} \mathrm{H}_{2} \mathrm{O}$, then flow rates would be threefold faster through the spiracle than the airways and fourfold faster through both pathways than through the airways alone.

In one emphysematous lung (lung 7) we measured the flowvolume relationship (fig 6A) and volume-time relationship (fig 6B) during passive deflation through the airways, through both spiracles simultaneously, and through both spiracles and the airway simultaneously. Flows and volumes were greater through both spiracles and airways together than with either pathway alone. This simulates the case of a living patient breathing through both spiracles and the tracheobronchial tree. The time constants via the bronchus and the spiracles were similar at $5.8 \mathrm{~s}$ and $5.2 \mathrm{~s}$, respectively, while the time constant emptying through all airways was $2.5 \mathrm{~s}$. This result might suggest that conductance through each pathway (bronchial and spiracle) is equal. This corroborates the graphic presentation in fig 6 and fits the electrical analogy as a capacitor discharging through two resistors in parallel.

It is reasonable to assume that, during passive deflation, both the bronchial tree and the spiracles share the same lung compliance-the capacitance of the model. If so, the driving pressure for flow through both pathways is the recoil pressure of the lung; any difference in flow between the two routes must be due to differences between Gaw and Gsp. Since the time constants in most of the emphysematous lungs were very similar by both routes, CL might have been greater when emptying through the spiracle than through the bronchial tree. This could be explained if the spiracles communicate with a greater volume of the lung parenchyma than the tracheobronchial tree.

This means that there were regions of trapped gas within the lung that communicate rapidly with the spiracles but do not communicate with open airways (or do so very slowly). As compliance is proportional to the total volume of communicating parenchyma, the lower resistance of the spiracle pathway would be counterbalanced by the higher compliance of the communicating parenchyma, so that the time constant of emptying via the spiracles could be similar to that via the airway. This would explain why more gas emptied via the spiracles than via the bronchial tree while the time course for emptying was almost identical for both pathways. The implication of this explanation is that gas trapping is not simply a phenomenon of small airways closing as RV is approached, but that the gas remains effectively trapped at nearly all lung volumes.

If these ideas are correct, the bronchial tree in emphysema does not communicate directly with all of the parenchyma except perhaps at volumes near TLC. The non-communicating parts presumably receive most of their gas from collateral channels. However, these channels must either be sufficiently slow so that emptying through them into the bronchial tree takes a longer time than is available during an expiration, or they must be closed at the lung volumes over which the expiration takes place. As a result, gas is effectively trapped with serious adverse effects on lung function. The amount of trapped gas that can escape from these regions via artificial airways through the pleural or bronchial wall ${ }^{5}$ is substantial and might lead to clinical benefit.

\section{Therapeutic implications}

The initial ${ }^{3} \mathrm{He}$ distribution in the control lungs was focal and irregular but generally similar via both the bronchial and spiracle routes. This probably relates to the fact that the tidal volume of $300 \mathrm{ml}$ was imposed on the lung through the spiracle. Being normal, the lung elastic properties were uniform. Thus, the ${ }^{3} \mathrm{He}$ would not remain localised to a region of parenchyma close to the spiracle, but would be forced through high resistance pathways to more central airways and then back down to the parenchyma subtended by these central airways. In emphysema, on the other hand, the ${ }^{3} \mathrm{He}$ was probably distributed by low resistance collateral channels, so there was no necessity for the gas to travel to central airways and then back down to the parenchyma. In any event, after a few breaths the ${ }^{3} \mathrm{He}$ distribution via airways and via spiracles was not strikingly different between the control and emphysematous lungs. Abnormalities in the distribution of ventilation in emphysema occurred over large macro regions on the scale of segments or even lobes. Within these regions the distribution was relatively homogeneous. ${ }^{3} \mathrm{He}$ distribution via spiracles tended to fill regions which were poorly ventilated by the bronchial tree even after several breaths. This further supports the evidence that the bronchial tree communicates poorly with large volumes of parenchyma that are accessed by spiracles. If breathing had taken place through both airways and spiracles simultaneously, the distribution would be similar to a superimposition of the left and right panels of fig 4. In all instances this would have provided a more even distribution of ${ }^{3} \mathrm{He}$ in emphysema.

The present data are in agreement with the results of Lausberg et $a l^{5}$ obtained by stent-supported fenestration through airway walls directly into the lung parenchyma in a control lung and 12 explanted emphysematous lungs inflated with $10 \mathrm{~cm} \mathrm{H}_{2} \mathrm{O}$ negative pressure in a chamber. Chamber pressure was rapidly converted to $20 \mathrm{~cm} \mathrm{H}_{2} \mathrm{O}$ positive pressure for measurement of the forced expiratory flow-volume curve. Forced expiratory curves in the control lung were not changed 
by placement of transbronchial stents but, in the emphysematous lungs, the mean (SD) $\mathrm{FEV}_{1}$ was 245 (107) ml before placement of the transbronchial stents ( $3 \mathrm{~mm}$ diameter), 447 (199) $\mathrm{ml}$ after three stents $(\mathrm{n}=12)$ and 666 (284) $\mathrm{ml}$ after five stents $(n=6)$. Similarly striking increases also occurred in total FVC in emphysematous lungs. Careful analysis of forced and passive expiration from full lung inflation in healthy subjects showed no difference in the rate of flow over most VC. ${ }^{6}$ The forced curves differed only by having an earlier onset of about 0.2 s. It may seem counterintuitive that the trapped gas RV of an emphysematous lung would be reduced equally well by small openings in either the bronchi or the pleura, but the data strongly support this. If the same trapped gas can be accessed both through the pleural surface and an airway, nonanatomical collateral channels must connect distant regions of trapped gas. It seems feasible to take advantage of this pathophysiology to develop effective treatment.

We conclude that supplementing the bronchial tree with additional parallel pathways to the lung parenchyma deserves further evaluation as a treatment for severe emphysema. Access to collateral ventilatory channels has the ability to diminish RV/ TLC, increase expiratory flow rates and VC, and improve distribution of ventilation. This technique may have therapeutic potential for relief of symptoms and improvement of lung function in patients with severe chronic obstructive pulmonary disease (GOLD stage 4).

\section{ACKNOWLEDGEMENTS}

The authors thank Drs G Alexander Patterson and Bryan F Meyers for their valuable support, Kathryn Cook and Diane Toeniskoetter for their technical assistance, Dawn Schuessler for editorial assistance, Dr David S Gierada for reviewing the radiographic data, Amersham Health (now General Electric) for the loan of a hyperpolariser apparatus and Broncus Technologies Inc (Mountain View, California, USA) for technical support.

\section{Authors' affiliations}

Cliff K Choong, Kimiaki Chino, Joel D Cooper, Division of Cardiothoracic Surgery, Washington University School of Medicine, St Louis, Missouri, USA

Peter T Macklem, Meakins-Christie Laboratories, Montreal Chest Institute, McGill University Health Centre Research Institute, Montreal, Quebec, Canada
John A Pierce, Stephen S Lefrak, Division of Pulmonary and Critical Care Medicine, Washington University School of Medicine, St Louis, Missouri, USA

Jason C Woods, Mark S Conradi, Department of Physics, Washington University, St Louis, Missouri, USA

Dimitry A Yablonskiy, Mallinckrodt Institute of Radiology, Washington University School of Medicine, St Louis, Missouri, USA

James C Hogg, McDonald Research Laboratories, St Paul's Hospital, University of British Columbia, Vancouver, British Columbia, Canada

Partial funding for this study was provided by NIH grant R01 HL62194.

Competing interests: None.

The Institutional Review Board for human studies approved the protocol (approval number 90-0510) and informed written consent was obtained from each subject.

\section{REFERENCES}

1 Hogg JC, Macklem PT, Thurlbeck WM. The resistance of collateral channels in excised human lungs. J Clin Invest 1969:48:421-31.

2 Terry PB, Traystman RJ, Newball HH, et al. Collateral ventilation in man. N Engl J Med 1978;298:10-5.

3 Morrell NW, Wignall BK, Biggs T, et al. Collateral ventilation and gas exchange in emphysema. Am J Respir Crit Care Med 1994;150:635-61.

4 Macklem PT. Collateral ventilation. N Engl J Med 1978;298:49-50.

5 Lausberg HF, Chino K, Patterson GA, et al. Bronchial fenestration improves expiratory flow in emphysematous human lungs. Ann Thorac Surg 2003;75:393-8.

6 Pierce JA. Studies of free collapse in the intact human lung. J Lab Clin Med 1959;54:96-106.

7 Saam BT, Yablonskiy DA, Kodibagkar VD, et al. MR imaging of diffusion of ${ }^{3} \mathrm{He}$ gas in healthy and diseased lungs. Magn Reson Med 2000;44:174-9.

8 Yablonskiy DA, Sukstanskii AL, LeaWoods JC, et al. Quantitative in vivo assessment of lung microstructure at the alveolar level with hyperpolarized ${ }^{3} \mathrm{He}$ diffusion MRI. Proc Natl Acad Sci 2002;99:31 11-6.

9 Dowdy S, Wearden S. Statistics for research. New York: John Wiley \& Sons, 1983:173-200.

10 Chino K, Pierce J, Cooper J, et al. Ventilation of excised human lungs via spiracles through the pleura. Am J Respir Crit Care Med 2003;167(Suppl):A546.

11 Laennec RTH. A treatise on diseases of the chest. Facsimile of the 1821 edition translated by John Forbes. Republished with permission 1962, Hafner: New York, 86.

12 Woolcock AJ, Macklem PT. Mechanical factors influencing collateral ventilation in human, dog, and pig lungs. J Appl Physiol 1971;30:99-115.

13 Higuchi T, Reed A, Oto T, et al. Relation of interlobar collaterals to radiologic heterogeneity in severe emphysema. Thorax 2006;61:409-13.

14 Cetti EJ, Moore AJ, Geddes DM. Collateral ventilation. Thorax 2006;61:371-3.

15 Woods JC, Yablonskiy DA, Choong Ck, et al. Long-range diffusion of hyperpolarized ${ }^{3} \mathrm{He}$ in explanted normal and emphysematous human lungs via magnetization tagging. J Appl Physiol 2005;99:1992-7.

\section{BNF for Children 2006, second annual edition}

In a single resource:

- guidance on drug management of common childhood conditions

- hands-on information on prescribing, monitoring and administering medicines to children

- comprehensive guidance covering neonates to adolescents

For more information please go to bnfc.org 